\title{
Cardiothoracic surgery in the midst of a pandemic: Operative outcomes and maintaining a coronavirus disease 2019 (COVID-19)-free environment
}

Damian Balmforth, FRCS(CTh), PhD, ${ }^{\mathrm{a}, \mathrm{b}}$ Martin T. Yates, MBBS, MD, ${ }^{\mathrm{a}}$ Kelvin Lau, FRCS (CTh), PhD, ${ }^{\mathrm{a}}$ Azhar Hussain, MBBS, ${ }^{a}$ Ana Lopez-Marco, FRCS (CTh), PhD, ${ }^{a}$ Stephen Edmondson, FRCS, (CTh), ${ }^{a}$ Aung Oo, FRCS (CTh), PhD, ${ }^{\mathrm{a}, \mathrm{b}}$ and Rakesh Uppal, FRCS (CTh), ${ }^{\mathrm{a}, \mathrm{b}}$ the Barts Cardiothoracic Research Collaborative*

\section{ABSTRACT}

Objective: In the United Kingdom, the coronavirus disease 2019 (COVID-19) pandemic has led to the cessation of elective surgery. However, there remains a need to provide urgent and emergency cardiac and thoracic surgery as well as to continue time-critical thoracic cancer surgery. This study describes our early experience of implementing a protocol to safely deliver major cardiac and thoracic surgery in the midst of the pandemic.

Methods: Data on all patients undergoing cardiothoracic surgery at a single tertiary referral center in London were prospectively collated during the first 7 weeks of lockdown in the United Kingdom. A comprehensive protocol was implemented to maintain a COVID-19-free environment including the preoperative screening of all patients, the use of full personal protective equipment in areas with aerosol-generating procedures, and separate treatment pathways for patients with and without the virus.

Results: A total of 156 patients underwent major cardiac and thoracic surgery over the study period. Operative mortality was $9 \%$ in the cardiac patients and $1.4 \%$ in thoracic patients. The preoperative COVID-19 protocol implemented resulted in 18 patients testing positive for COVID-19 infection and 13 patients having their surgery delayed. No patients who were negative for COVID-19 infection on preoperative screening tested positive postoperatively. However, 1 thoracic patient tested positive on intraoperative bronchoalveolar lavage.

Conclusions: Our early experience demonstrates that it is possible to perform major cardiac and thoracic surgery with low operative mortality and zero development of postoperative COVID-19 infection. (JTCVS Open 2020;4:107-14)

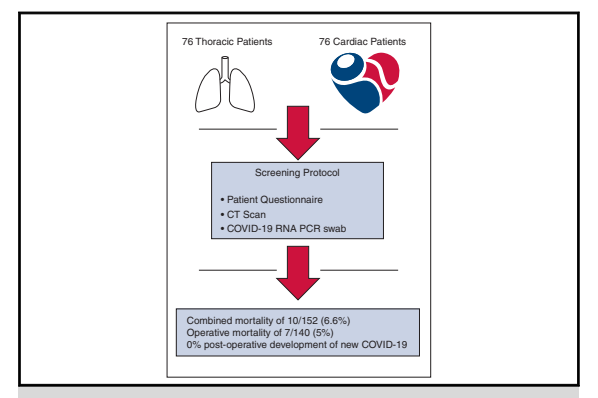

Summary of screening protocols used and operative outcomes during the COVID-19 pandemic.

CENTRAL MESSAGE

It is possible to perform major cardiac and thoracic surgery in

the midst of the COVID-19

pandemic with low operative

mortality and zero development

of postoperative COVID-19

infection.

\section{PERSPECTIVE}

The COVID-19 pandemic presents unique challenges to the provision of cardiothoracic surgery globally. We describe our early experience of operating during the pandemic, our success in maintaining a COVID-19-free environment, and operative outcomes during the first 7 weeks of the United Kingdom's lockdown. Patient pathways for outpatients, interhospital transfers, and emergency surgery are discussed.

See Commentary on page 115 .

\footnotetext{
From the a'Department of Cardiothoracic Surgery, St Bartholomew's Hospital; and ${ }^{\mathrm{b}}$ Queen Mary University of London, London, United Kingdom.

* Barts Cardiothoracic Research Collaborative: A. Sepehripour, K. Lall; N. Roberts; C. Di Salvo; S. Kolvekar; K. Wong; S. Ambekar; A. Sheikh; B. Adams; J. Yap; D. Lawrence; W. Awad; A. Shipolini; C. Rathwell; Mohamed Rahnavardi, Steven Stamenkovic, David Waller, Henrietta Wilson, May Al-Sahaf.

Received for publication Sept 7, 2020; accepted for publication Sept 18, 2020; available ahead of print Oct 22, 2020.
}

Address for reprints: Rakesh Uppal, FRCS (CTh), Department of Cardiothoracic Surgery, St Bartholomew's Hospital, West Smithfield, London, EC1A 7BE United Kingdom (E-mail: rakesh.uppal@nhs.net).

2666-2736

Copyright (C) 2020 The Authors. Published by Elsevier Inc. on behalf of The American Association for Thoracic Surgery. This is an open access article under the CC BY-NCND license (http://creativecommons.org/licenses/by-nc-nd/4.0/).

https://doi.org/10.1016/j.xjon.2020.09.003 


\section{Abbreviations and Acronyms \\ BAL = bronchoalveolar lavage \\ COVID-19 = coronavirus disease 19 \\ $\mathrm{CT}=$ computed tomography \\ ITU = intensive therapy unit \\ $\mathrm{LDH} \quad=$ lactate dehydrogenase \\ PPE $\quad=$ personal protective equipment}

The coronavirus disease 2019 (COVID-19) pandemic presents unique challenges to the provision of cardiothoracic surgery globally. In response to the high demand for ventilators and intensive therapy unit (ITU) beds created by the virus, the United Kingdom ceased all elective surgical procedures. However, there remains a need to provide cardiac surgery for urgent and emergency cases as well as timecritical thoracic cancer surgery. In London, where the prevalence of COVID-19 cases in the United Kingdom was greatest, we met this need by introducing a strict protocol to maintain a COVID-19-free environment. This was mandated by early reports of high mortality associated with developing COVID-19 postoperatively. ${ }^{1,2}$

This protocol consisted of preoperative COVID-19 screening for all patients, the use of full personal protective equipment (PPE) in areas with aerosol-generating procedures, behavioral measures to minimize inadvertent patient-to-patient and staff-to-patient transmission, and the designation of separate treatment floors within the hospital for patients with and without the virus. The inception of this protocol coincided with the United Kingdom being placed into lockdown. Similar protocols have been described in the current literature. ${ }^{3-5}$ At present, there are no published reports of outcomes for major cardiac or thoracic surgery performed during the pandemic.

The aim of this paper is to describe our early experience of operating during the pandemic. We report our ability to maintain a COVID-19-free environment and the operative outcomes during the first 7 weeks of lockdown. The different patient pathways for outpatients, interhospital transfers, and emergency surgery are discussed.

\section{METHODS}

All patients undergoing major cardiac or thoracic surgery between March 26, 2020, and May 10, 2020, at a single institution were prospectively entered into a database. In addition to the routine collection of preoperative demographics, data were collected on the results of COVID-19 screening tests both pre- and postoperatively, as well as patient outcomes including in-hospital mortality and discharge destination. All data were collected as part of an ongoing audit of surgical provision in the COVID19 pandemic and anonymized before analysis. Approval was granted by the local institutional review board.

Patients undergoing cardiac or thoracic surgery followed tailored pathways aimed at maintaining a COVID-19-free environment in dedicated ITUs and wards. A detailed overview of our protocol has been published, ${ }^{6}$ and a simplified version of the patient pathways and screening protocols is shown in Figure 1. In brief, all patients requiring interhospital transfer for urgent or emergency surgery were screened at the time of referral with a screening questionnaire and upper respiratory tract swab for COVID-19 RNA. If patients were asymptomatic and swab negative, they were transferred to our institution and kept in isolation until a second swab confirmed they were negative for COVID-19. Following these 2 negative swabs, patients underwent computed tomography (CT) of the chest to exclude signs of COVID-19 pneumonia as well as blood test including lymphocyte count (usually $<1$ in active infection) and lactate dehydrogenase (LDH) (usually $>300$ in active infection). Provided that a patient is asymptomatic, swab negative for COVID-19, has no evidence of COVID-19 infection on CT, and screening blood tests are within normal limits, then surgery was scheduled. If patients were suspected of being positive COVID-19 on screening, they were immediately transferred to wards dedicated to the treatment of patients with COVID-19.

Patients admitted from home for surgery were advised to self-isolate at home for 2 weeks before being admitted to a side room on a dedicated COVID-19-free ward. They then underwent the same aforementioned screening protocol. Patients who tested positive in this cohort were discharged home and their operation was postponed for at least 30 days to allow lung recovery.

At the time of surgery, all patients underwent a deep tracheal aspirate/ bronchoalveolar lavage (BAL) on which a COVID-19 RNA polymerase chain reaction was performed. If the BAL was positive, patients were transferred to intensive care units or wards dedicated to the treatment of patients with COVID-19. Postoperative swabs were not performed routinely but at the discretion of the treating physician in response to persistent fever, cough, or clinical or radiologic features of pneumonia.

Alongside the patient screening protocol, we implemented measures to minimize traffic on the ward. We reduced the number of doctors attending patients to a minimum and grouped patients by specialty to minimize medical staff traveling between wards. All medical and non-medical staff including cleaning and catering staff were required to wear facemasks at all times in clinical areas in line with national guidance. Medical staff were not routinely screened for COVID-19 infection but were isolated and swabbed if they developed symptoms.

All surgery was performed using full PPE using FFP3 masks. In unscreened patients, visors were worn over loupes. Hoods were worn for unscreened patients undergoing rigid bronchoscopy or extended pleurectomy decortication. Personnel in theater were limited to a minimum and theaters were deep cleaned between patients. Postoperatively, patients were treated in ring-fenced COVID-19-free intensive therapy units. Staff wore full PPE at all times while in the ITU. No visitors are allowed in the hospital for the duration of the pandemic.

\section{RESULTS}

An overview of the baseline characteristics, screening test results, and operative outcomes for both cardiac and thoracic surgery is shown in Table 1.

\section{Operative Outcomes}

During the study period, 76 patients were admitted for cardiac surgery. Of these, $56(73.7 \%)$ were inpatients requiring urgent surgery, $16(21 \%)$ were emergency admissions, and just $4(5.3 \%)$ were elective patients admitted from home. In total, $67(88 \%)$ patients underwent surgery as planned. Of the remaining 9 patients, 3 were emergencies who died before reaching the operating theater, 1 was discharged home as they were not deemed in need of surgery during their admission, and 5 tested positive for 


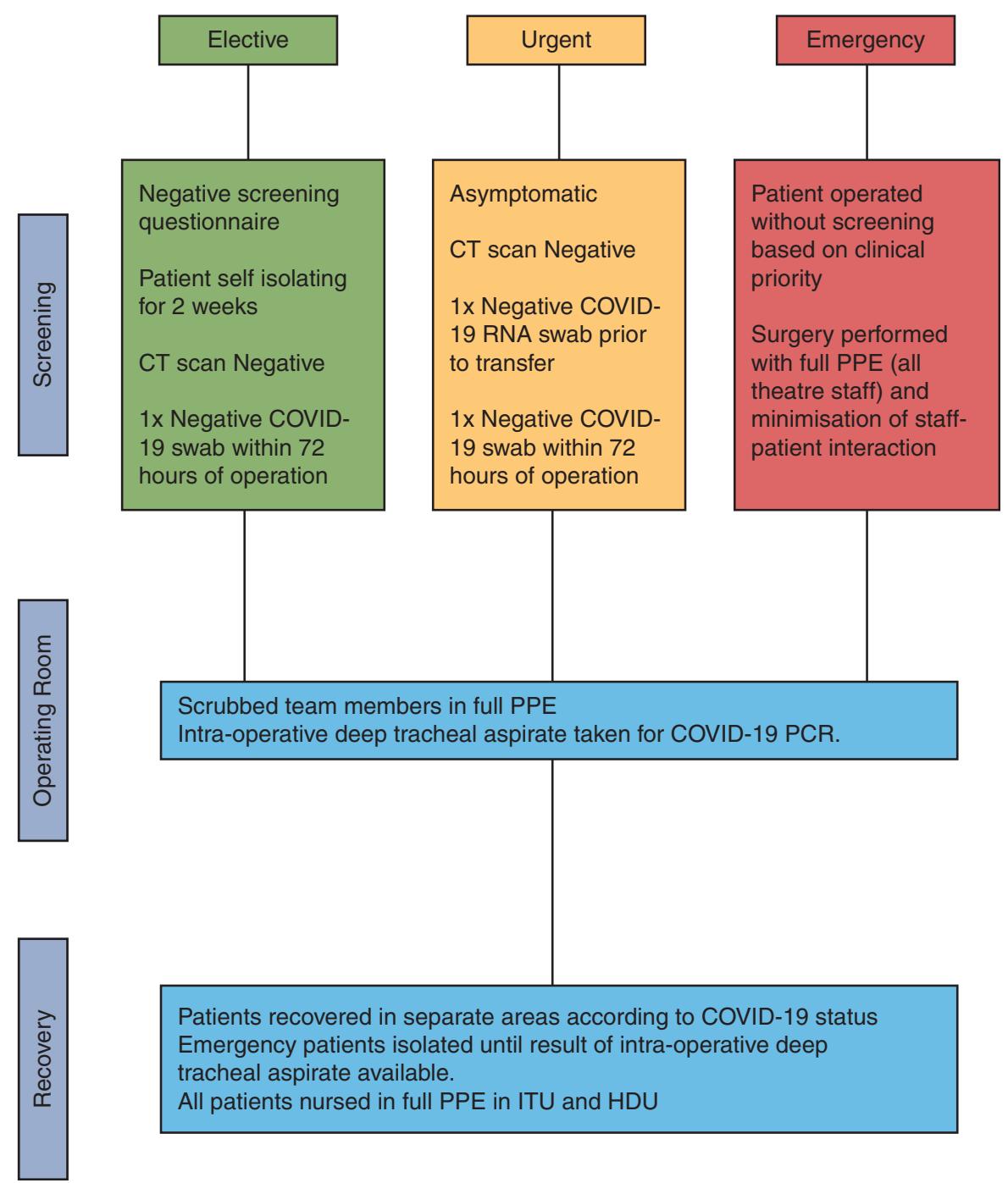

FIGURE 1. An overview of the patient pathways for undergoing major cardiac and thoracic surgery at our institution. $C T$, Computed tomography; COVID-19, coronavirus disease 2019; PPE, personal protective equipment; PCR, polymerase chain reaction; ITU, intensive therapy unit; HDU, highdependency unit.

COVID-19 and were discharged home to recover. The types of operations performed on the 67 patients who had surgery are shown in Figure 2, A.

Of the 76 patients planned for cardiac surgery, 61 $(80.2 \%)$ were discharged home. The median postoperative stay was 6 days. Nine patients $(11.8 \%)$ died during their admission with an operative mortality of 6 of $67(9 \%)$. This is comparable with the mean European System for Cardiac Operative Risk Evaluation II for the operated population of $6.3 \%$. Four of these mortalities were emergency patients; 3 type A aortic dissection repairs and 1 decompensated acute mitral regurgitation. The remaining 2 were highrisk urgent operations; 1 third time redo descending thoracic aortic aneurysm (endoleak) and 1 acute aortic infective endocarditis (embolic phenomena).
During the same period, 76 patients were planned for thoracic surgery. Of these, $66(87 \%)$ were elective admissions from home, $9(11.8 \%)$ were urgent inpatients, and 1 $(1.3 \%)$ was an emergency. In total, $73(96 \%)$ underwent surgery with 1 patient cancelled preoperatively due to progression of disease and 2 patients discharged home after testing positive for COVID-19 infection. The operations performed (Figure 2, $B$ ) covered the full breadth of thoracic surgery, including 1 extended pleurectomy-decortication, 3 bronchoplastic lobectomies, 1 lobectomy with chest-wall resection-reconstruction, 11 segmentectomies, 18 roboticassisted operations, 1 transsternal thymectomy for thymomatous myasthenia gravis, and 1 airway stenting. A wide range of high-risk patients were operated including an 89and a 92-year-old patient both undergoing video-assisted 
TABLE 1. Overview of baseline characteristics, screening test results, and operative outcomes for cardiac and thoracic surgery

\begin{tabular}{|c|c|c|}
\hline & Cardiac surgery & Thoracic surgery \\
\hline Total planned surgeries & 76 & 76 \\
\hline Male sex & 56 & 45 \\
\hline Average age, y $( \pm \mathrm{SD})$ & $62( \pm 11.6)$ & $63( \pm 15)$ \\
\hline \multicolumn{3}{|l|}{ Operative urgency } \\
\hline Elective & 4 & 66 \\
\hline Urgent inpatient & 56 & 9 \\
\hline Emergency & 16 & 1 \\
\hline \multicolumn{3}{|l|}{ Preoperative management } \\
\hline Surgery & 67 & 73 \\
\hline Discharged due to COVID-19 & 5 & 2 \\
\hline Discharged—clinical & 1 & 1 \\
\hline Died preoperatively & 3 & 0 \\
\hline \multicolumn{3}{|l|}{ Screening } \\
\hline Preoperative COVID-19 swab and CT & 54 & 68 \\
\hline Preoperative swab only & 12 & 4 \\
\hline Preoperative CT only & 10 (all type A dissection) & 2 \\
\hline No Preoperative COVID screening (swab or CT) & 0 & 2 \\
\hline Patients COVID positive on prescreening & 15 & 3 \\
\hline Swab and CT positive & 4 & 0 \\
\hline Swab positive only & 7 & 2 \\
\hline CT positive only & 4 & 1 \\
\hline Number of intraoperative conversions on BAL & 0 & 2 \\
\hline Number of postoperative conversions on throat swab & 0 & 0 \\
\hline Total number of patients in whom surgery delayed due to COVID-19 positive & 11 & 2 \\
\hline \multicolumn{3}{|l|}{ Outcomes } \\
\hline \multicolumn{3}{|l|}{ Discharge outcome } \\
\hline Discharged home & 61 & 73 \\
\hline Discharged other & 5 & 2 \\
\hline Remain inpatient & 1 & 0 \\
\hline Dead & 9 & 1 \\
\hline Median postoperative stay, $\mathrm{d}$ & 6 & 4 \\
\hline Operative mortality & $6 / 67(9 \%)$ & $1 / 73(1.4 \%)$ \\
\hline
\end{tabular}

$S D$, Standard deviation; COVID-19, coronavirus disease 2019; $C T$, computed tomography; $B A L$, bronchoalveolar lavage.

thoracic surgery for lobectomy, and a patient with heart failure and inducible ischemia due to a blocked left anterior descending stent undergoing robotic-assisted lobectomy.

Of the 76 patients planned for thoracic surgery, $73(96 \%)$ were discharged home. The median postoperative stay was 4 days. One patient $(1.3 \%)$ died during their admission, giving an operative mortality of 1 of $73(1.4 \%)$. This patient died from disease progression following drainage of a malignant pleural effusion.

\section{Screening Outcomes}

Of the 76 cardiac patients, $54(71 \%)$ underwent the full screening protocol with both COVID-19 swabs and CT of the chest. Ten patients who did not have a preoperative swab were type A aortic dissections, where the need to perform emergency surgery precluded waiting the minimum 24 hours required to process the swab result. Of the 13 patients who did not have a CT of the chest, 4 were operated on the first day of the study when the protocol for CT scanning had not yet been introduced, 4 were discharged due to positive swab results, and 4 were deemed too unstable to have a CT scan. In total, 15 patients were identified as positive for COVID-19 on preoperative screening; 7 on swab alone, 4 with CT alone, and 4 with both swab and CT. Of these 15,11 had their surgery deferred to allow time to clear the virus, with 5 being discharged home and 6 staying for inpatient surgery. All 6 of these patients survived to discharge. As such, delaying transfer or surgery due to positive COVID screening was not associated with poor outcomes. Of the remaining 4 patients who tested positive for COVID-19 on screening, 1 died preoperatively from their heart condition despite being transferred for emergency surgery, 2 patients proceeded with surgery without delay, and 1 patient tested positive while on the operating table. The patients who were operated without delay were deemed too unstable to defer their 
surgery despite their COVID-19 status. The patient who tested positive on the operating table was during the first day of the protocol and had had an extra swab sent after initially screening as negative just 24 hours before. The 3 operated patients who were positive for COVID-19 recovered in clinical areas dedicated to patients with COVID-19, with staff wearing full PPE. All 3 patients survived and were discharged home. In the operated group, no previously negative patients tested positive for COVID-19 infection on intraoperative BAL swab or postoperative upper respiratory tract swab.

For thoracic surgery patients, 68 patients $(89 \%)$ were screened for COVID-19 preoperatively with upper respiratory tract swab and CT Chest. Four patients had COVID-19 swab but no CT, 2 patients had CT but no swab, and 2 patients were not screened preoperatively. These 2 patients were operated on the first day that the protocol was introduced. In the thoracic group, 2 asymptomatic patients had surgery delayed after positive COVID-19 screens preoperatively, 1 due to a positive swab and 1 due to suspicious CT findings. One patient who tested positive on preoperative swab proceeded with surgery without delay on clinical grounds, as he was septic from empyema. One patient screened as negative preoperatively subsequently tested positive on BAL. This patient had a history on COVID-19 infection 2 weeks before her operation and had returned 4 negative preoperative swabs. Both of these patients recovered on wards dedicated for the treatment of patients with COVID-19 and were discharged without developing COVID-19 pneumonitis. No previously negative patients tested positive on postoperative upper respiratory tract swabs.

In the combined cohorts, 5 patients ( 1 thoracic and 4 cardiac) were classified as positive for COVID-19 on CT alone in the presence of negative swab results. Of these, 2 cardiac patients proceeded to surgery without delay on clinical grounds. The remaining 3 had their surgery delayed. All 3 were subsequently operated on after returning further negative swab and remaining asymptomatic for COVID-19 infection.

Figure 3 shows the mean values for lymphocyte and LDH for patients who were positive and negative for COVID-19. There is no correlation between serum markers and COVID-19 positivity in this cohort. Mean lymphocyte count in the 17 patients positive for COVID-19 in combined cardiac and thoracic cohort was $1.7\left(\times 10^{9} / \mathrm{L}\right)$ compared with 1.9 in patients not positive for COVID-19 $(P=.380)$. Mean LDH level in patients positive for COVID-19 was 306 units/L compared with 213 in patients who were negative $(P=.068)$.

\section{DISCUSSION}

The COVID-19 pandemic has presented a global challenge to the delivery of cardiothoracic surgery. The few studies that are available to date on operative outcomes in the setting of COVID-19 infection report high mortality. Lei and colleagues ${ }^{1}$ report on a series of 34 elective operations across a range of specialties from Wuhan, China, performed at the start of the pandemic with an overall mortality of $20.5 \%$. In a case series of 7 patients who developed COVID-19 infection in the perioperative period of major lung resection, the mortality was $42.9 \%{ }^{2}$ These findings mirror our own experience of operating before implementing the screening protocol described here. During this period, 9 patients developed COVID-19 pneumonia postoperatively, with a mortality of $44 \%{ }^{7}$ As such, protocols for delivering safe surgery during the pandemic are crucial. In recent weeks, several groups have reported protocols and guidance for the safe delivery of cardiac surgery during the pandemic. ${ }^{3-5}$ Little literature is available describing practical experience of implementing them or the outcomes that result. The current report describes our early experience of performing major cardiac and thoracic surgery in the midst of the pandemic and focuses on 2 main issues: (1) how to provide good surgical outcomes at a time when resources are deployed elsewhere and (2) how to maintain a COVID-19-free environment in which to operate.

At our institution 2400 cardiac surgical operations, 1500 thoracic operations, 3000 percutaneous coronary interventions, and 400 transcatheter valve implantations are performed annually. During the study period, 67 urgent and emergency cardiac procedures and 73 thoracic procedures were performed compared with 149 and 195 procedures for the same period in 2019. The primary reason for the reduced case number was the reduction in resources available to the department due to staff and ITU beds being reallocated to the treatment of COVID-19. As such, some case selection had to be undertaken to prioritize these reduced resources. Lower-risk patients for whom it was thought safe to defer surgery were discharged home, resulting in a higherrisk operative cohort than before the pandemic. Despite the high-risk nature of the patient cohorts in this study, we did not observe significantly greater death rates than expected. The cardiac operative mortality of $9 \%$ is not significantly greater than the $4 \%$ mortality observed in the 149 urgent and emergency patients operated between March and April $2019(P=.07)$ and compares with a predicated mortality of $6.3 \%$ from the cohort's predicted mean European System for Cardiac Operative Risk Evaluation II. Of the 6 cardiac patients, 4 were emergency operations and 2 were highrisk urgent operations (leaking descending thoracic aneurysm and infective endocarditis). Our reported cardiac mortality should be viewed in the context that the operated cohort was greater risk than our usual practice. During the study period, the agreed criteria for conventional surgical intervention for ischemic heart disease was modified with a bias toward percutaneous coronary intervention and early 


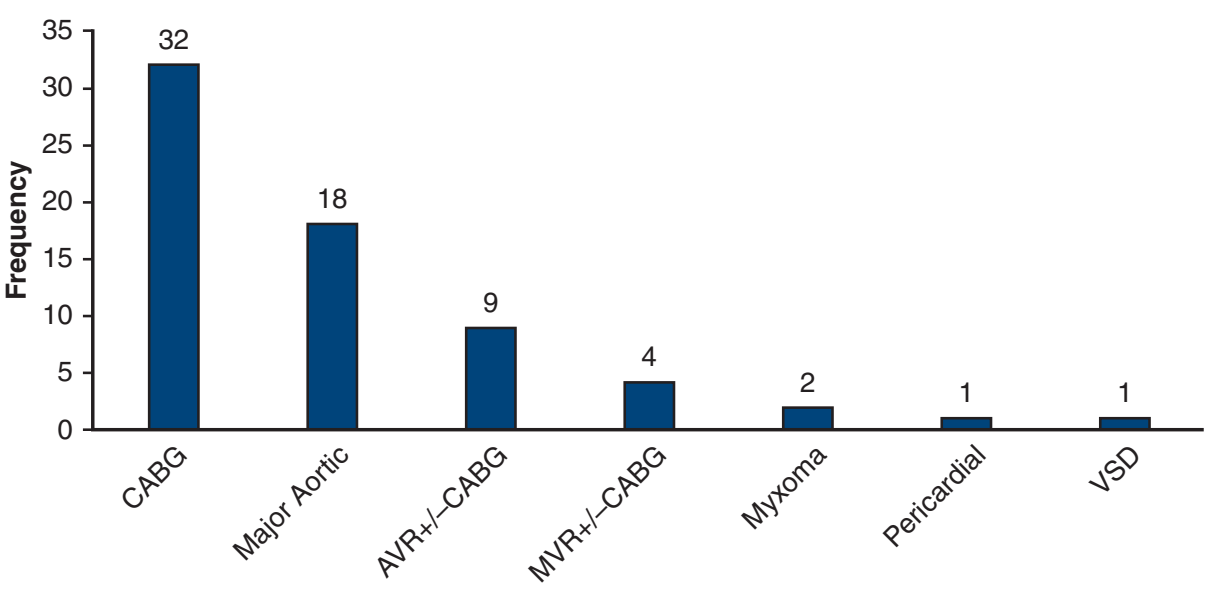

A

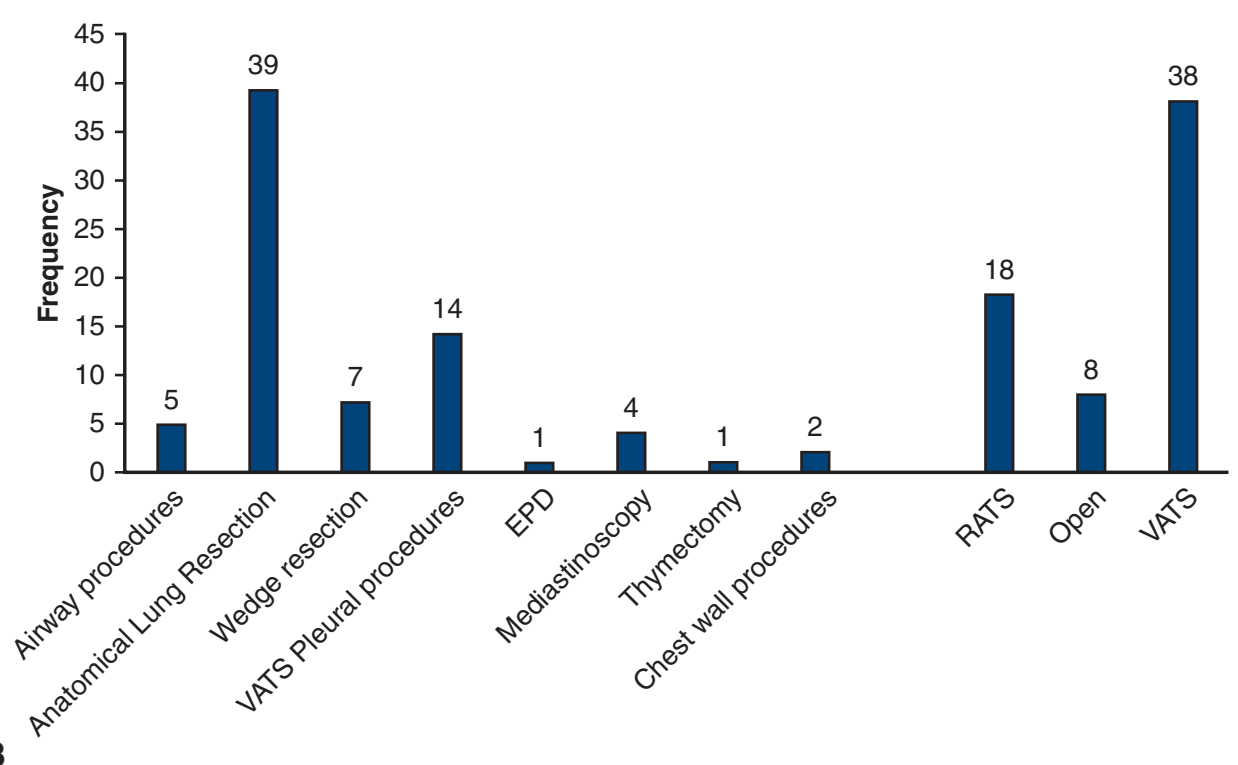

FIGURE 2. Overview of the types and volume of operations performed during the COVID-19 pandemic. A, Cardiac surgical operations. B, Thoracic surgical operations and approaches used. $C A B G$, Coronary artery bypass grafts; $A V R$, aortic valve replacement; $M V R$, mitral valve replacement; VSD, ventricular septal defect; VATS, video-assisted thoracic surgery; $E P D$, extended pleurectomy decortication; RATS, robot-assisted thoracic surgery.

discharge to a safe environment. This was agreed nationally between the governing cardiac surgical and interventional cardiology societies. As such, the threshold for patients requiring inpatient surgery during the pandemic was raised due to the additional risk associated with remaining in hospital.

In addition to the cardiac surgical results, we observed excellent operative mortality of just $1.4 \%$ in 73 thoracic operations. These data confirm that our ability to perform major cardiac and thoracic procedures safely has not been diminished by the pandemic.

From the January 1, 2020, to the May 13, 2020, there were 1996 patients admitted with confirmed COVID-19 infection to our trust. ${ }^{8}$ Of these, 361 patients were admitted to the intensive care unit, 281 required mechanical ventilation, and 19 were treated with extracorporeal membrane oxygenation. The current report demonstrates that the protocol implemented to isolate, screen, and cohort patients according to their COVID-19 status has been successful in maintaining a COVID-19-free environment across the wide range of patients undergoing surgery. The combination of patient isolation and 2 upper respiratory tract RNA swabs were able to distinguish COVID-19 infection. We found that the addition of a CT scan did not ultimately identify any cases of COVID-19 infection over and above the RNA swab. All 5 patients diagnosed as positive for COVID-19 on CT with negative swabs were subsequently shown not to have COVID-19 infection. As such, we have now removed the preoperative CT scan from our preoperative screening protocol. This is in line with recent joint guidance from the Royal College of Radiologists and the Royal College of Surgeons. The inclusion of lymphocytes and 

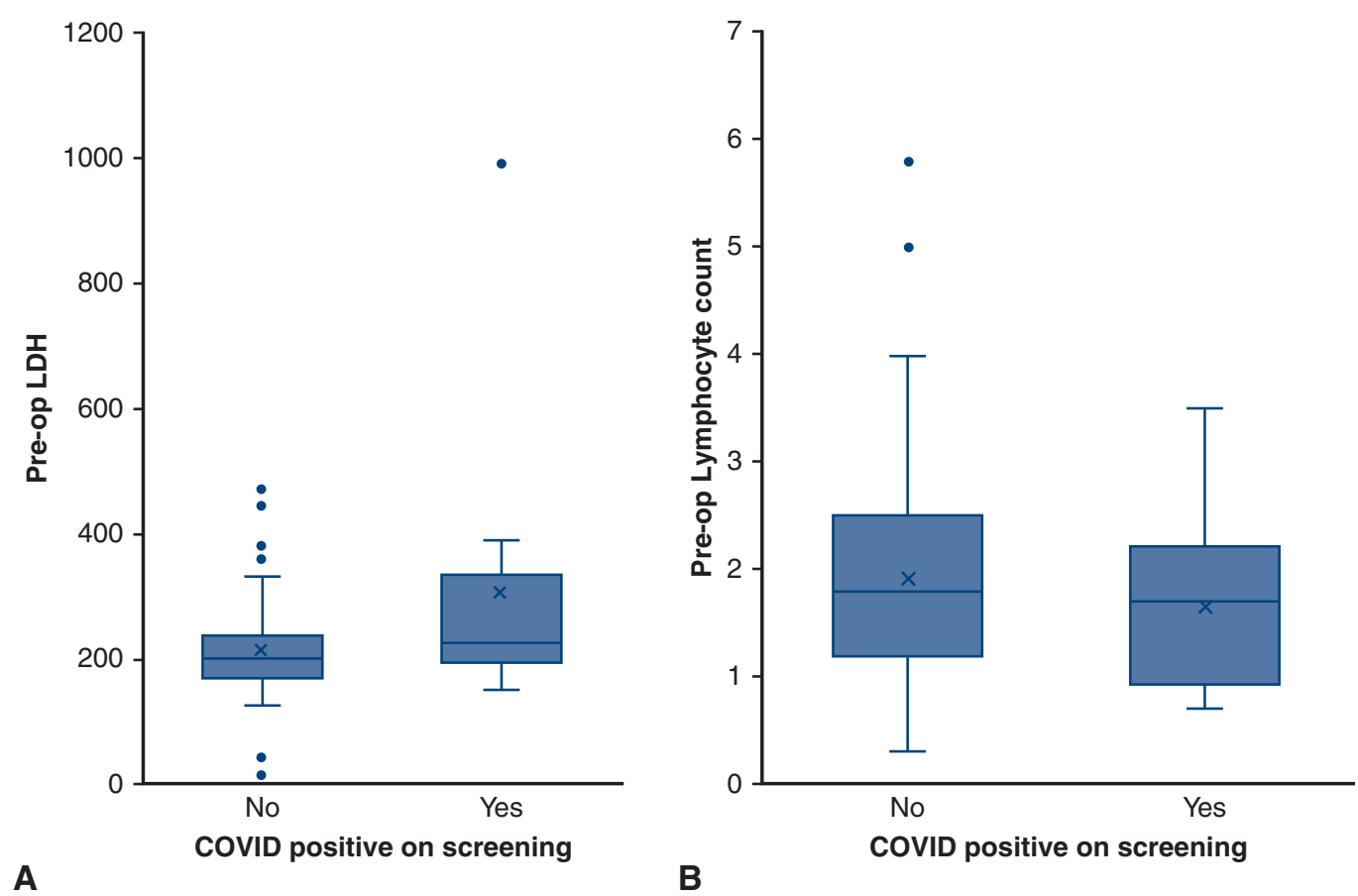

FIGURE 3. Box and whisker plots showing a comparison of (A) LDH levels and (B) lymphocyte count in patients with and without COVID-19 infection. No significant differences were seen for either lymphocyte count $(P=.380)$ or $\mathrm{LDH}(P=.068)$. The middle horizontal line represents the median whereas the upper and lower borders of the box represent the upper and lower quartiles. The upper and lower whiskers represent the maximum and minimum values of non-outliers. Extra dots represent outliers. LDH, Lactic acid dehydrogenase; COVID-19, coronavirus disease 2019.

LDH levels in the screening protocol made a negligible impact on decision making. Our analysis of these tests in the 156 patients in this cohort found no significant difference between the levels of either lymphocytes or LDH in the presence of COVID-19 infection. LDH in particular was often ignored entirely, and there were frequent occurrences of hemolysis leading to an untestable sample $(\mathrm{n}=24,15.4 \%)$. As such, we do not recommend the use of serum tests in the screening of COVID-19 infection.

The cardiac and thoracic surgical cohorts presented here represent very different populations of patients. The cardiac cohort comprised acutely unwell patients, with the vast majority being urgent and emergency cases. These patients were often admitted from other hospitals, which are areas of high COVID-19 prevalence. By contrast, the thoracic cohort were primarily elective patients being admitted from home. Their surgery continued to be performed in the midst of the pandemic due to the negative outcomes associated with delaying diagnosis and treatment in cancer patients. However, many of these patients were high-risk patients at risk of the severe form of COVID-19 due to their comorbidities and pre-existing lung conditions. Despite these differences, the implemented protocol was $100 \%$ successful in preventing patients contracting the virus postoperatively. There was 1 thoracic case that returned a positive swab from BAL samples taken intraoperatively.
Swabs taken from BAL have been shown to be the gold standard test for COVID-19 infection, ${ }^{9}$ and this highlights the false-negative rate of the upper airway swabs ${ }^{9}$ and the importance of not concluding a patient has cleared the virus just because the throat swab is negative. This false-negative rate is the rationale for performing BAL on all operated patients. As a result of the implemented screening program, 11 patients in the cardiac cohort and 2 patients in the thoracic cohort had their surgery deferred to allow time for lung recovery. No emergency operations were delayed due to the screening program. If no COVID-19 swab result was available, surgery continued using full PPE. Postoperatively, the patient was nursed in isolation until the result of the intraoperative BAL sample was available.

In the cardiac cohort, the number of patients who were positive on preoperative screening was greater than the thoracic cohort (15/76 [19.7\%] vs 3/76 [3.9\%]). One explanation is that the cardiac patients were predominantly acute admissions from other hospitals whereas the thoracic patients had been isolating at home for a minimum of 2 weeks before admission. Recent evidence suggests that the incidence of COVID-19 infection both among health care workers and in the community has reduced significantly since the onset of lockdown. ${ }^{10}$ This, together with the low rate of COVID-19 positivity in the elective cohorts, prompted a decision to offer cardiac surgery to patients at home 
who were known to be developing worsening symptoms. As the pandemic progressed, 4 patients have been admitted from home for cardiac surgery after 2 weeks of isolation. As the prevalence of COVID-19 falls, operations will resume for the backlog of patients for whom surgery has been deferred. When considering operating on this lower risk group of patients, it is important to be able to provide a COVID-19-free environment as well as no additional operative mortality. We plan to use the pathways described in this report to progressively increase the volume of patients undergoing elective cardiac and thoracic surgery. We believe that the success of this pathway has provided the route back to full capacity operating in the pandemic.

\section{CONCLUSIONS}

Our early experience demonstrates that it is possible to perform major cardiac and thoracic surgery with low operative mortality and zero development of postoperative COVID-19 infection.

\section{Conflict of Interest Statement}

The authors reported no conflicts of interest.

The Journal policy requires editors and reviewers to disclose conflicts of interest and to decline handling or reviewing manuscripts for which they may have a conflict of interest. The editors and reviewers of this article have no conflicts of interest.

\section{References}

1. Lei S, Jiang F, Su W, Chen C, Chen J, Mei W, et al. Clinical characteristics and outcomes of patients undergoing surgeries during the incubation period of COVID-19 infection. EClinicalMedicine. 2020;23:100385.

2. Cai Y, Hao Z, Gao Y, Ping W, Wang Q, Peng S, et al. Coronavirus disease 2019 in the perioperative period of lung resection: a brief report from a single thoracic surgery department in Wuhan, People's Republic of China. J Thorac Oncol. 2020;15:1065-72.

3. Engelman DT, Lother S, George I, Funk DJ, Ailawadi G, Atluri P, et al. Adult cardiac surgery and the COVID-19 pandemic: aggressive infection mitigation strategies are necessary in the operating room and surgical recovery. Ann Thorac Surg. 2020;110:707-11.

4. Haft JW, Atluri P, Alawadi G, Engelman D, Grant MC, Hassan A, et al. Adult cardiac surgery during the COVID-19 pandemic: a tiered patient triage guidance statement. Ann Thorac Surg. 2020;160:452-5.

5. Hassan A, Arora RC, Adams C, Bouchard D, Cook R, Gunning D, et al. Cardiac surgery in Canada during the COVID-19 pandemic: a guidance statement from the Canadian Society of Cardiac Surgeons. Can J Cardiol. 2020;36:952-5.

6. Hussain A, Balmforth D, Yates M, Lopez-Marco A, Rathwell C, Lambourne J, et al. The Pan London Emergency Cardiac Surgery service: coordinating a response to the COVID-19 pandemic. J Card Surg. 2020;35:1563-9.

7. Yates MT, Balmforth D, Lopez-Marco A, Uppal R, Oo AY. Outcomes of patients diagnosed with COVID-19 in the early postoperative period following cardiac surgery. Interact Cardiovasc Thorac Surg. 2020.

8. Apea VJ, Wan YI, Dhairyawan R, Puthucheary ZA, Pearse RM, Orkin CM, et al. Ethnicity and outcomes in patients hospitalised with COVID-19 infection in East London: an observational cohort study. medRxiv. 2020:2020.06.10.20127621.

9. Wang W, Xu Y, Gao R, Lu R, Han K, Wu G, et al. Detection of SARS-CoV-2 in different types of clinical specimens. JAMA. 2020;323:1843-4.

10. Treibel TA, Manisty C, Burton M, McKnight Á, Lambourne J, Augusto JB, et al. COVID-19: PCR screening of asymptomatic health-care workers at London hospital. Lancet. 2020;395:1608-10.

Key Words: COVID-19, cardiothoracic Surgery, outcomes, pandemic 